\title{
Novel model for defining electricity tariffs using residential load profile characterisation
}

\author{
Sima Davarzani ${ }^{1}$, Ioana Pisica ${ }^{1}$ and Laurentiu \\ Lipan $^{2}$
}

\author{
${ }^{1}$ Brunel University London, Electronic and Computer Engineering \\ Department, Kingston Lane, Uxbridge, UB8 3PH, UK \\ ${ }^{2}$ University Politehnica of Bucharest, Electric Power Systems \\ Department, Splaiul Independentei 313, Bucharest, Romania
}

Keywords: Demand response; electricity market; electricity tariffs; price elasticity; residential load profile

\begin{abstract}
In recent years, great attention towards enabling future smart grid functionalities in distribution networks creates significant changes in electricity load profiles. These changes require a new advanced paradigm in electricity pricing and tariffs definition aiming to increase the potential of responsiveness demand. This paper aims to propose a new model for designing residential electricity tariffs using their load profile clustering and characterization, taking into account social, technical and educational factors alongside the financial incentives. The methodology is based on estimation of the potential of demand responsiveness to dynamic pricing over time for different cluster of households with similar electricity consumption patterns. In this regard, different time of use pricing bands has been considered and compared with fixed price tariffs. Moreover, a baseline demand for each cluster of households has been determined based on the elasticity of demand to different static tariffs. The results show that the proposed model can provide distribution network operators (DNOs) and suppliers with a basic and general outline of defining more effective dynamic pricing schemes that reflect intermittent nature of residential demand.
\end{abstract}

\section{Introduction}

The revolution of smart meters provides a smarter electricity network which improves the reliability, safety, efficiency and asset utilization of the power network [1]. The introduction and availability of such an advanced and automated infrastructure technologies in distribution system 
create an alternation in the future energy system. The new concept of active network provides new opportunities for electricity customers to participate in demand reductions schemes and therefore causes additional roles for the grid [2]. This can be possible through the introduction of several tariffs to the market aiming to increase the level of responsiveness demands.

The design and modelling of an electricity market generally aim to enhance the economic benefits while maintaining both customers and suppliers' satisfaction. However, it is important to note that the level of responsiveness to such programs significantly impacts on implementation of various tariffs and pricing structures with regards to decreasing electricity bills through peak demand reduction programs [3].

The demand side management potential and effectiveness of pricebased Demand Response (DR) techniques have been analysed in some researches [4-6]. Moreover, several dynamic tariffs have been introduced and implemented for residential electricity customers in order to enhance the efficiency of DR programs and improve the operational issues at power networks [7-9]. These programs are generally categorised as incentive based or price-based programs [10]. Price-based programs such as Time of Use (ToU) Pricing, Critical Peak Pricing (CPP) or Real Time Pricing (RTP) aim to change the customer's electricity behaviour through offering various pricing bands over time. Customers can benefit from lower prices at non-peak time or receive some incentives for either load shedding or load shifting during specific DR event period. However, each group of customers can experience different level of financial satisfaction due to their unique and distinguished characteristics.

This paper presents a comprehensive approach aiming to design more efficient electricity tariffs through studying electricity behaviour characterisation of residential customers. The main idea of this approach is based on clustering customers into well-defined, nonoverlapping classes where each cluster is ranked with specific potential of responsiveness and band to changes in prices over time.

For this purpose, a characteristic-based clustering has been applied to a dataset including more than 3500 Irish households with half hourly meter readings. For each cluster of households, probability and potential of both load shifting as well as load shedding during different periods of time has been assessed. Then, each cluster has been ranked based on the elasticity of demand to different ToU tariffs along with their attitudes towards engagement in DR programs. In addition, a baseline demand profile has 
been allocated to each cluster of customers for switching between different tariffs.

The remainder of the paper is structured as follows: Methodology of the proposed approach is elaborated in section 2 which presents the designing of electricity tariffs for different group of customers based on potential of demand responsiveness and baseline demand in each group. Section 3 describes the case studies and data initialization. Results are analysed and discussed in detail in Section 5 and finally the conclusions and future research are presented in Section 6.

\section{Methodology}

\subsection{Potential of demand responsiveness}

Generally, the aim of demand response programs can be categorised as load shedding which refers to overall load reduction overt time and load shifting which encourages customers to shift their power usage to nonpeak periods. We have used the proposed methodologies in [11] and [12] in order to find the probability of shifting/shedding demand and consequently the available DR from each cluster of customers during a typical day.

In this regard, the load profiles of electricity customers of each case study have been clustered with K-means algorithm for one month in July. Therefore, the potential of DR for each cluster $\left(P_{D R, C}\right)$ has been calculated as:

$$
P_{D R, c}=\sum_{i=1}^{N c}\left(P_{A}-P_{E}\right)
$$

Where $P_{A}$ and $P_{E}$ denote the actual and responsiveness demand (estimated demand after DR) respectively which arising from $N c$ customers in cluster $c . P_{E}$ depends on the type of DR schemes which can be formulated by equation 2 and 3 in order to estimate the total potential of curtailment and shiftable demands from all aggregated loads within each cluster. 


$$
\begin{aligned}
& P_{E}=\sum_{i=1}^{N c} \int_{t=t s}^{t=t e}\left(P_{h, \max }(i, t)-P_{h, \min }(i, t)\right) d t \\
& P_{E}=\sum_{i=1}^{N c} \int_{t=t s}^{t=t e}\left(P_{h, \max }(i, t)-P_{B}(i, t)\right) d t
\end{aligned}
$$

Where, $P_{h, \max }$ and $P_{h, \min }$ are the maximum and minimum power consumption which has been calculated based on the historical data for $i^{\text {th }}$ customer in $c^{\text {th }}$ cluster over studied time periods $\left(t_{s}-t_{e}\right) . P_{B}$ is the shiftable demand level which has been described in the next subchapter.

\subsection{Baseline demand}

The baseline demand can be defined as the minimum level of power consumption from aggregated of demands in each cluster. Therefore, the updated demand profile after employing a price signal $\left(P_{n e w, D R, c}\right)$ can be determined as the difference between actual $\left(P_{A}\right)$ and baseline demand $\left(P_{B}\right)$ and estimated as:

$$
P_{n e w, D R, c}=\sum_{i=1}^{N c}\left(P_{A}-P_{B}\right)
$$

It should be noted that $\mathrm{P}_{\mathrm{B}}$ is related to not only the potential of DR but also to a set of household's characteristics $\left(Q_{i}\right)$ and attitudes of customers towards participating in DR programs $\left(A_{i}\right)$ as:

$$
P_{B}=\sum_{i=1}^{N c} f\left(P_{D R, c}, A_{i}, Q_{i}\right)
$$

$P_{D R, C}$ is the potential of the maximum available from aggregation of all $N_{i}$ associated shiftable demands in cluster $C$ and can be modelled by equation 6:

$$
\mathrm{P}_{\mathrm{DR}, \mathrm{c}}=\sum_{\mathrm{i}=1}^{\mathrm{Ni}}\left(\mathrm{P}_{\mathrm{DR}, \mathrm{c}}(\mathrm{i})\right)
$$

The classification of each household has been considered in customer clustering process. As a result, the effects of different features have been included in calculating the potential of DR, $Q_{i}$ has been ignored in equation 3 for the simplicity purpose. 
Novel model for defining electricity tariffs using residential load profile characterisation

\subsection{Defining Electricity tariff}

The granularity of demand responsiveness $(P)$ to different price signal $(C)$ can be measured by the price elasticity of demand factor $(E d)$ as:

$$
E_{d}=\frac{\mathrm{P}}{C_{d}} \times \frac{\mathrm{d} C_{d}}{\mathrm{dP}}=\left\{\begin{array}{cc}
E_{d}<1 & \text { Inelastic } \\
E_{d}>1 & \text { Elastic }
\end{array}\right.
$$

The ToU tariffs are designed to increase the motivation of electricity customers in reducing/shifting demand especially during peak times in the aim of power balancing at the distribution network. In order to gain more effective tariffs, an aggregation of the minimum incentives and baseline demand of each cluster of customers should be optimised subject to systems constraints and customers comfortable and satisfaction level. Therefore, the effect of different ToU tariffs on the overall demand reduction during peak times is the interest of this study. In this regard, for each group of customers, the relationship between different pricing bands with the demand elasticity has been analysed with respect to the baseline demand.

\section{Case Studies}

This paper studies the dataset related to The Ireland Electricity Smart Metering Trails (IEMST) which consists of more than 3900 Irish [13] households with half hourly meter readings. An in-home survey was also available and has been investigated along with their load profiles in order to characterise the residential electricity consumption patterns and behaviours. In this study, we have considered the data related to one summer month in July. Therefore, customers with more than one day missing value in their meter readings have been removed.

After cleaning and clearing data, 3996 of customers have been remained and analysed in this study. Dataset consists of a six month benchmark period (July- Dec 2009) and one year trial period (Jan-Dec 2010). The smart meters were installed and power usage of households was measured during benchmark period with fixed price tariffs. In the trial period, customers have been grouped to remain in their normal (fixed) tariff called controlled group or to switch from their current tariff to different ToU 
tariffs and DSM stimuli monitoring scenarios called ToU group. Table 1 shows the tariffs structures and population allocated in each tariff.

\section{Clustering results}

Characterising the residential customers based on their power usage behaviour is an effective tool in order to deal with their intermittent and uncertain nature. The results of clustering can provide different groups of customers with similar potential of DR.

The clustering results have been calculated based on the K-means algorithm using Euclidean distances. The optimal number of clusters has been determined by Davies-Bouldin Index (DBI).

First, customers have been grouped based on their general prospective towards DR participating (Table 3). Then, a new clustering has been applied on the customers with only highly motivated attitudes (3690 customers). In this regard, the estimated potential of overall power reduction obtained from each individual household during benchmark period has been computed. Moreover, clustering has been analyzed for weekdays in July. Finally, each cluster has been classified in terms of households and occupancies characteristics using multinomial regression analysis. The regression model analyses the correlations between dependent variables (customer's characterization) and mean usage during the period of test studies within each cluster. Table 2 and Table 3 describe the achieved results and the properties of each cluster for both case studies. In Table 3, the main features which can have an effect on the power usage have been grouped and analysed. The household feature relates to the general features of the household such as size of household and number of bedrooms.

Table 1. Tariffs scenarios and poulation in IESMT trial [13]

\begin{tabular}{|c|c|c|c|c|c|c|}
\hline \multirow{2}{*}{ Price band } & \multicolumn{6}{|c|}{ Price by tariff group (c/Kwh) } \\
\cline { 2 - 7 } & $\boldsymbol{A}$ & $\boldsymbol{B}$ & $\boldsymbol{C}$ & $\boldsymbol{D}$ & $\boldsymbol{W}$ & Control \\
\hline Night (23:00 to 08:00) & 12 & 11 & 10 & 9 & 10 & \\
\hline Day (All other times) & 14 & 13.5 & 13 & 12.5 & 14 & 18 \\
\hline Peak (17:00 to 19:00, Mon to Fri) & 20 & 26 & 32 & 38 & 38 & \\
\hline weekend (All Weekend) & & & & & 16 & \\
\hline Population & 1368 & 511 & 1370 & 509 & 100 & 1170 \\
\hline
\end{tabular}


Novel model for defining electricity tariffs using residential load profile characterisation

TABLE 2. Customers clustering based on average power consumption

\begin{tabular}{|c|c|c|c|c|c|c|}
\hline \multirow{2}{*}{$\begin{array}{c}\text { Cluster } \\
\text { No. }\end{array}$} & \multirow{2}{*}{$\begin{array}{c}\text { Population } \\
(\%)\end{array}$} & \multirow{2}{*}{$\begin{array}{c}\text { Mean } \\
\text { usage }\end{array}$} & \multicolumn{2}{|c|}{$\begin{array}{c}\text { Potential of reduction } \\
(\mathbf{K w})\end{array}$} & \multicolumn{2}{|c|}{$\begin{array}{c}\text { Potential of reduction } \\
\text { (overall) }\end{array}$} \\
\cline { 4 - 7 } & & & Weekday & Weekends & Weekday & Weekends \\
\hline 1 & 22.84 & 360.35 & 1314.42 & 840.55 & 1910.88 & 1333.34 \\
\hline 2 & 22.71 & 362.80 & 577.87 & 364.28 & 824.01 & 546.15 \\
\hline 3 & 25.63 & 394.10 & 1073.83 & 680.56 & 1546.45 & 1062.46 \\
\hline 4 & 3.25 & 55.75 & 343.69 & 219.60 & 465.25 & 324.44 \\
\hline 5 & 12.65 & 197.45 & 979.30 & 630.14 & 1395.94 & 960.70 \\
\hline 6 & 12.89 & 200.32 & 63.46 & 114.12 & 82.31 & 170.97 \\
\hline
\end{tabular}

TABLE 3. Customers charactrisation within each cluster

\begin{tabular}{|c|c|c|c|c|c|c|}
\hline $\begin{array}{c}\text { Cluster } \\
\text { No. }\end{array}$ & $\begin{array}{c}\text { Priority } \\
\text { based on } \\
\text { DR } \\
\text { potential }\end{array}$ & $\begin{array}{c}\text { Highly } \\
\text { motivated } \\
(\%)\end{array}$ & $\begin{array}{c}\text { Household } \\
\text { feature }\end{array}$ & $\begin{array}{c}\text { Occupancy } \\
\text { level }\end{array}$ & $\begin{array}{c}\text { Educational } \\
\text { level }\end{array}$ & $\begin{array}{c}\text { Economic } \\
\text { level }\end{array}$ \\
\hline 1 & 1 & 80.42 & 6.842898 & 3.834268 & 6.512359 & 4.584632 \\
\hline 2 & 4 & 78.70 & 4.573384 & 1.992343 & 4.635458 & 3.401898 \\
\hline 3 & 2 & 78.57 & 4.822042 & 2.147352 & 5.115433 & 2.812393 \\
\hline 4 & 5 & 84.50 & 1.839991 & 3.472032 & 6.397739 & 4.031594 \\
\hline 5 & 3 & 79.94 & 1.839991 & 1.216999 & 1.904661 & 1.425251 \\
\hline 6 & 6 & 77.16 & -1.57424 & -0.15855 & -1.71991 & -1.46396 \\
\hline
\end{tabular}

It should be noted that the population distribution in different ToU tariffs follows a normal distribution in terms of household's characteristics. Therefore, the potential of either demand reduction or demand shifting is calculated in each cluster regarding to the same proportion of households characteristics within each group (Fig.1).

Comparing Table 2 and Table 3, it can be vividly perceived that in spite of high percentage of DR participating interest among the population in all clusters, the potential of DR does not follow an equal proportion regarding the population within each group. It can be illustrated as the divergent customer's characteristics in terms of the level of contentment, convenience, awareness as well as financial aspects.

In addition, the resulted regression coefficients show that the ranking of clusters based on estimated potential of DR not only depends on power 
usage but also individual characteristics of each household. For instance, cluster 6 comprises of customers with quite high mean electricity

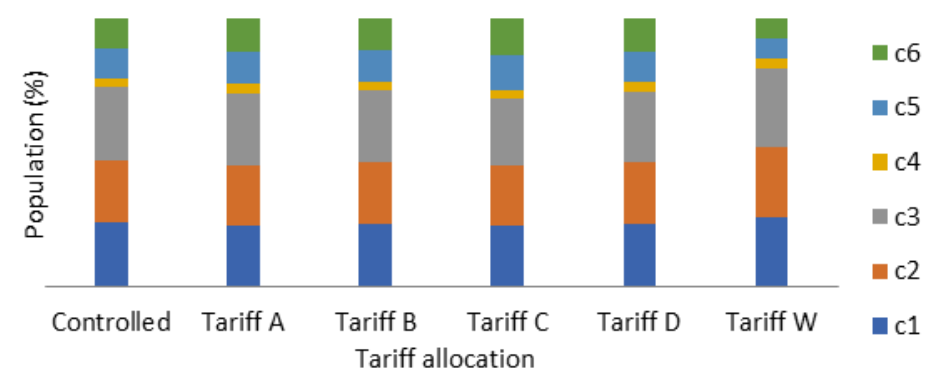

Fig. 1. Distribution of population based on tariffs allocation within each group

consumption compared to cluster 4 , but the potential of DR is less.

\section{Results and discussion}

This section summarises the achieved results for opportunities from customers of the case studies to participate in DR programs. The main interest and objective are to investigate the relation between the estimated DR potential with the actual DR resulted after trial period.

Fig.2 shows the achieved results of estimating the DR potential (from benchmark period) and actual DR (from trial period) during a typical day (48 timeslot) with aggregating demands in all weekdays (Mon-Fri excluding bank holidays) on July. Moreover, the average minimum and maximum power usage from aggregation of demands within each cluster have been presented. The calculated DR is based on overall demand reduction from all households regardless of tariff allocation.

The probable available DR from each cluster is estimated with quite high perception. Regarding to the fact that it is inevitable that there is always a percentage of uncertainty due to the inconstant customer's electricity behaviour, the curve of load profiles before and after applying DR follow a similar trend. Besides, the difference between the two DR profile can also occur because of the relatively temporal condition such as weather temperature.

The overall consumption before DR (Fixed price) and after DR (different ToU scenarios) is shown in Fig. 3. In other words, it explains the price elasticity of demand to changes in electricity prices depending on the 

characterisation

time of day. As can be seen, each group has a distinctive and similar pricing band for changing their consumption pattern. This can be highly relative to the financial and occupancy factors of each group. For instance, cluster 1 and 2 are less elastic to low band pricing as they refer to more wealthy and educated groups. The effects of pricing are also different for weekdays and weekends on different clusters.

The correlation between each tariff type as dependent and overal DR as independent parameter within each cluster has been calculated with linear regression model and has been drawn in Fig. 4. It describes the relation and effects of different ToU tariffs on different cluster of households. Accordingly, it can be used as a guideline towards designing dynamic tariffs and pricing bands which depends on the network topology as well as the requirement of demand curtailment.
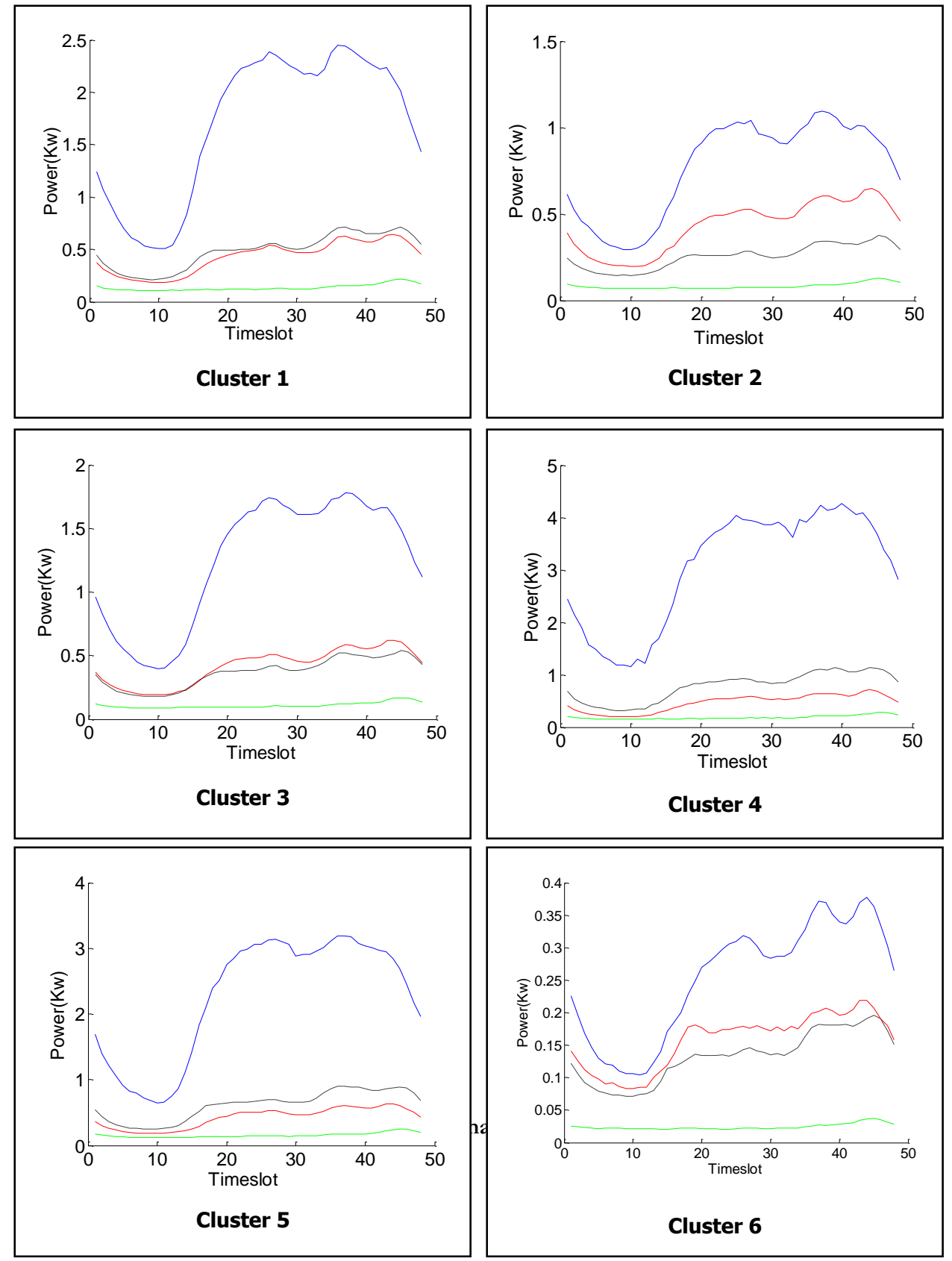

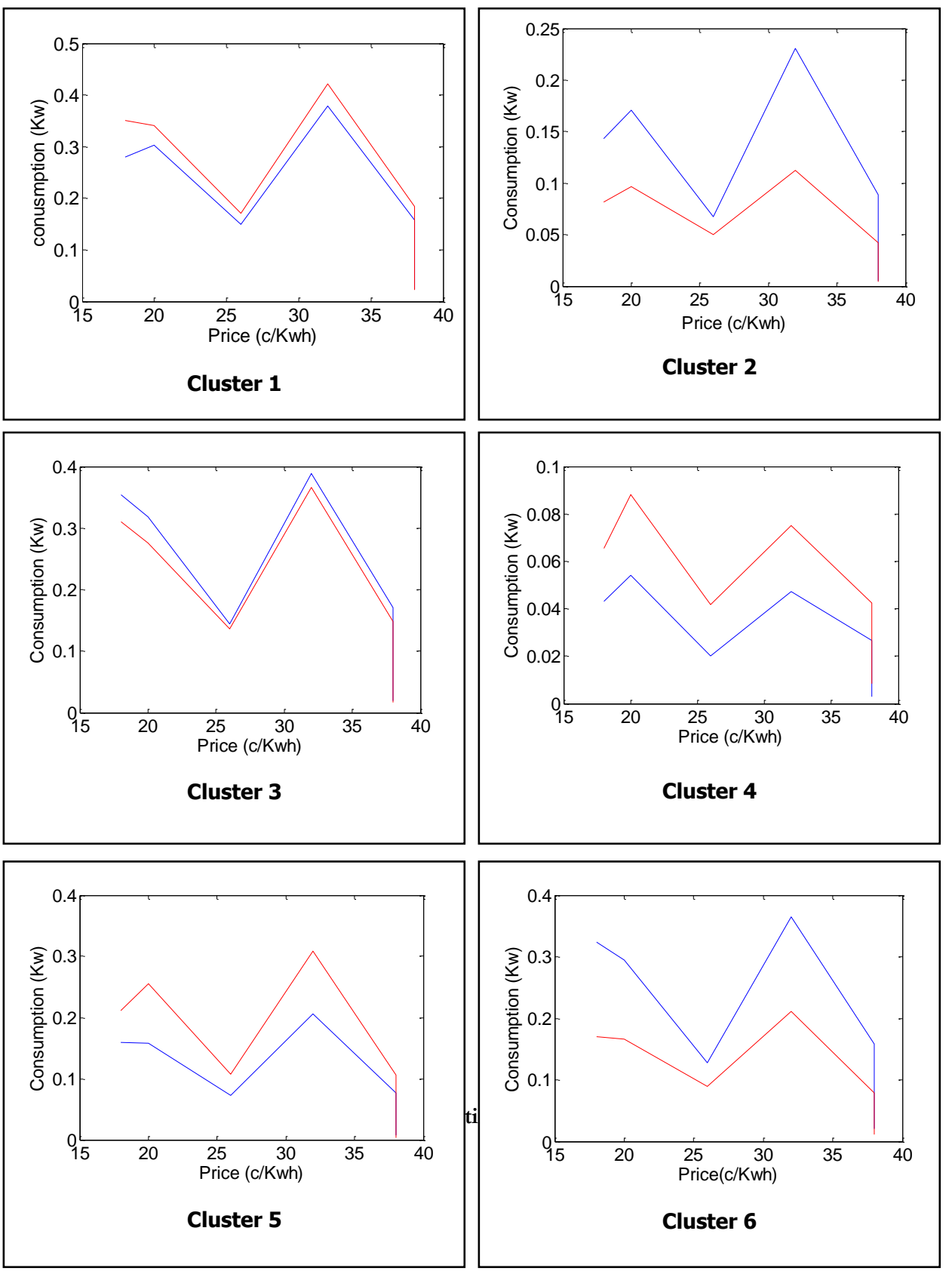

Mean power usage before DR

Mean power usage after DR 


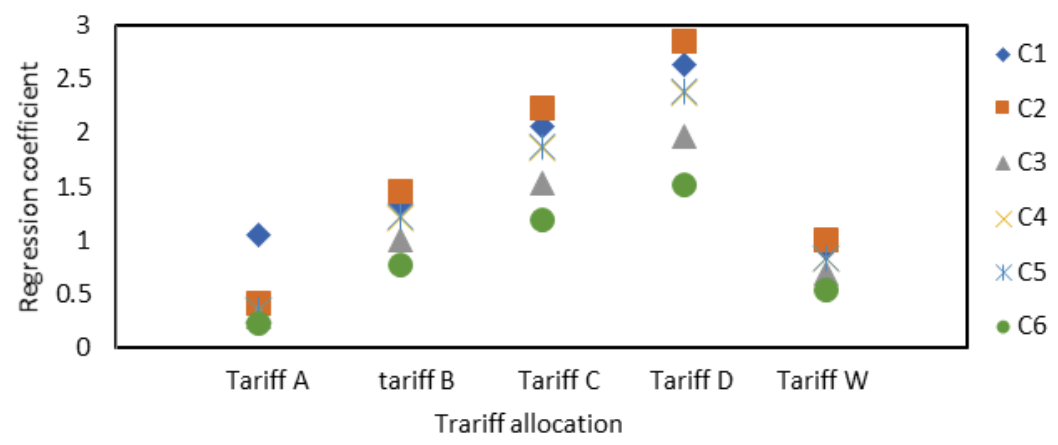

Fig. 4. Regression coefficients for different tariffs allocation and different groups of customers

\section{Conclusion and future work}

This paper assesses the potential of residential responsiveness demand under different ToU tariffs. The aim is to present a new model and a guideline towards designing more effective dynamic pricing. This has been done through predicting available DR from different group of households with similar electricity patterns and characteristic feature. The estimated DR has been compared with actual DR achieved from trial experiment. A baseline demand has been calculated using price elasticity of demand as well as linear regression analysis.

The results show that the demand elasticity to price varies based on specific tariff ratio. This is due to the different potential of demand reduction level, characteristics and relative financial benefits in each cluster of customers. The prediction of potential DR is relatively compatible with the actual results. In addition, all customers are elastic to ToU tariffs, though the economic benefits vary based on the actual available level of DR potential.

Future research will include the implementation of the proposed methodology in a typical distribution network aiming to improve the equilibrium between demand and supply. Moreover, dynamic pricing (near real time) is another point of interest which can be combined with distribution automation in end-use systems in order to deliver more benefits to both network operators and consumers.

\section{References}


[1] Telefonica, "The Smart Meter Revolution" https://iot.telefonica.com/system/files_force/the_smart_meter_revoluti on_brave_new_world_5.pdf?download=1, Accessed: Jan 2017.

[2] World Economic Forum, "The future of electricity new technologies transforming the grid edge," http://www3.weforum.org/docs/WEF_Future_of_Electricity_2017.pdf, Accessed: March 2017.

[3] "Load Profiles and their use in Electricity Settlement," https://www.elexon.co.uk/wpcontent/uploads/2013/11/load_profiles_v2.0_cgi.pdf, Accessed Nov. 2014.

[4] C. Bartusch, K. Alvehag, "Further Exploring the Potential of Residential Demand Response Programs in Electricity Distribution," Applied Energy, pp. 39-59, 2014.

[5] T. Ericson, "Households' self-selection of dynamic electricity tariffs," Applied Energy, pp.2541-7, 2011.

[6] R. Stamminger and V. Anstett, "The Effect of Variable Electricity Tariffs in the Household on Usage of Household Appliances," Smart Grid and Renewable Energy, vol. 4, pp. 353-365, 2013.

[7] T. Nguyen, H.T. Nguyen, and L. B. Le, "Dynamic Pricing Design for Demand Response Integration in Power Distribution Networks," IEEE Transaction on Power Systems, vol. 31, no. 5, pp. 3457- 3470, Sept. 2016.

[8] D. Steen, L. A. Tuan and O. Carlson, "Effects of Network Tariffs on Residential Distribution Systems and Price-Responsive Customers Under Hourly Electricity Pricing," IEEE Transaction on Smart Grid, vol. 7, no. 2, pp. 617- 626, March. 2016.

[9] J. Xiao, J. Y. Chung, J. Li, R. Boutaba and J. W. Hong, "Near Optimal Demand-Side Energy Management Under Real-time DemandResponse Pricing," POSTECH University, Pohang, South Korea, 2010.

[10] H. T. Haider, O. H. See and W. Elmenreich, "A Review of Residential Demands Response of Smart Grid, "Renewable and Sustainable Energy Reviews, vol. 56, pp. 166-178, 2016.

[11] S. Davarzani, I. Pisica and G. Taylor, "Development of a Novel Multi-Agent System for Residential Voltage Control Using Demand Response based on Customer Behaviour," IEEE International Conference on Innovative Smart Grid Technologies (IEEE ISGT Europe), Torino, Italy, Sept. 2017. (Accepted April 2017).

[12] S. Davarzani, I. Pisica, G. Taylor, "A Novel Methodology for Predicting Potential Responsiveness in Residential Demand", International Conference on Resilience of Transmission and 
Novel model for defining electricity tariffs using residential load profile characterisation

Distribution Networks (RTDN), Birmingham, UK, Sept. 2017. (Accepted 15 June 2017).

[13] Irish Social Science Data Archive. Data from the Commission for Energy Regulation (CER) - smart metering project. http://www.ucd.ie/issda/data/commissionforenergyregulationcer/; Accessed Feb. 2017. 